\title{
POTENCIAL PESQUERO Y ESTUDIOS ECOLOGICOS DE BAHIA MAGDALENA I. LAS EXISTENCIAS DE CAMARON CON REFERENCIA ESPECIAL AL CAMARON DE ROCA O CAMARON JAPONES.
}

\author{
por: \\ Christopher Paul Mathews \\ y \\ Jorge Arturo González \\ Ciencias Marinas Vol. 2 Núm. I
}

\begin{abstract}
RESUMEN
Se presentan datos sobre la distribución, la abundancia, la tasa de crecimiento y el ciclo de vida de Scyonia sp. en Bahía Magdalena, B. C. Sur. Se concluye que puede vivir hasta 20-30 meses; propbablemente desova entre mayo y agosto. En las muestras predominan las hembras, y únicamente son las hembras los individuos mayores de $8 \mathrm{~cm}$ de longitud total.

ABSTRACT

Data on the distribution, abundance, growth rates and life cycle of Scyonia sp. are presented for the stock found in Magdalena Bay, B. C. Sur. Scyonia sp. may live up to 20-30 months long in this area, and probable spawns sometime between the end of March and the beginning of August. Females predominate in the catches, and all specimens are over $8 \mathrm{~cm}$ in total length are females.
\end{abstract}

Para la elaboración de este trabajo se recibió la ayuda de los pescadores de la cooperativa Isla Macapule de Guasave, Sinaloa, además fue presentado como ponencia en el $V$ Congreso Nacional de Oceanografía celebrado en Guaymas, Son., en octubre de 1974.

\section{METODOS}

Los métodos de conversión de los datos de las capturas a abundancias $\mathrm{kg} / \mathrm{ha}$ se presentan en otra parte de esta publicación (Mathews y Espinoza). Para el camarón se asumió arbitrariamente un valor de 1.0 para la vulnerabilidad, como es habitual para las capturas de peneidos (Berry, 1967; Gotshall, 1972). Se efectua- ron dos cruceros, el primero del 7-20 de febrero y el segundo del 20-27 de agosto, 1974, en la zona de Bahía Magdalena.

Se midieron algunas muestras del camarón $1 \mathrm{~mm}$ de longitud total, pero los datos se juntaron al $0.5 \mathrm{~cm}$ puesto que hubo un problema con las mediciones milimétricas: los medidores demostraron una tendencia para preferir los valores conteniendo la unidad $(70,80,90 \mathrm{~mm}$ etc.). Juntando los datos al $0.5 \mathrm{~cm}$ se' elimina esta fuente de error.

La mayoria de las mediciones se efectuaron hasta el $0.5 \mathrm{~cm}$ (o al $\mathrm{mm}$ ) más bajo o sea los individuos fr $1.5-1-9 \mathrm{~cm}$. se agrupan en la clase de $1.5 \mathrm{~cm}$.; $\mathrm{fr}$ 2.0-2.4, en la clase $2.0 \mathrm{~cm}$., etc. 
Se sexaron camarones en algunas muestras $y$ se pesaron en el laboratorio camarones congelados en febrero $y$ se pesaran frescos a bordo del barco, en agosto.

\section{RESULTADOS}

\section{Comparación de las capturas.}

Se capturaron individuas de tres especies, el camarón azul, Penaeus stylirostris, el camarón café, Penaeus californiensis $y$ el camarón de roca Scyonia sp. Las dos primeras especies son comerciales desde hace muchos años, mientras que la última comienza a entrar en los mercados. Su tamaño pequeño y su carapacho duro, han impedido que ascienda a los mismos precios que las otras especies, pero su excelente sabor deberá asegurarle un lugar permanente en el mercado. Puesto que esta última especie es la más desconocida y la más importante en Bahía Magdalena, se considera aquí su biología en detalle.

\section{DISTRIBUCION GEOGRAFICA}

En la Fig. I presentamos los datos sobre la distribución geográfica del camarón de roca en la zona de $\mathrm{Ba}-$ hía Magdalena. En primer lugar se nota que está casi totalmente ausente de Bahía Almejas, en invierno tanto como en verano. En segundo lugar notamos que existe una zona cercana a la costa oriental de Isla Magdalena donde se encuentra un centro de población del camarón de roca que persiste durante los dos cruceros. Esta zona coincide generalmente con la zona más profunda de la Bahía y especialmente con una zona donde parece existir una surgencia permanente de aguas frías (Alvarez Borrego y Galindo Bect, no publicadol. Esta zona se considera como el centro permanente del camarón de roca en Bahía Magdalena.

El camarón de roca en agosto tiene una distribución muy irregular afuera de la zona de aguas profundas y frías; los datos para febrero no son completos: la distribución del camarón de roca era tan extensa en la Bahía, que no se pudo controlar la existencia con la atención de- bida sin ignorar a las otras especies. Pero se puede decir que el camarón de roca fue la especie dominante en las comunidades demersales en febrero mientras que en agosto fuera de la zona central de distribución, fue irregular. La Fig. 1 es incompleta en cuanto a la distribución invernal del camarón de roca. En la Fig. 2 presentamos los datos sobre la distribución batimétrica de la abundancia del camarón de roca, en organismos/ha. Se nota una variación extremada en las abundancias que suben hasta casi 4,000 /ha. y que bajan hasta menos de 50/ha. Estas abundancias corresponden a niveles de $2-10 \mathrm{~kg} / \mathrm{ha}$ de ca-. marón de roca, con valores más altos en lances excepcionales. A pesar de que existen diferencias entre las abundancias diurnas y nocturnas, no se notan tales diferencias entre las abundancias invernales y de verano. Las abundancias promedios en invierno y en verano son de 1099 y 852 respectivamente.

\section{CRECIMIENTO}

En la Fig. 3 se presentan los datos gráficos de la frecuencia y de las tallas de los camarones de roca capturados en febrero y en agosto. Se nota que el tamaño mödal en febrero fue de $5.5 \mathrm{~cm}$ $y$ de $7.0 \mathrm{~cm}$ con muchos individuos de $7-8 \mathrm{~cm}$ en agosto. Las mediciones de los individuos más grandes en agosto llegan a $13.0 \mathrm{~cm}$ y en febrero hasta $10.5 \mathrm{~cm}$ solamente. De esto se puede deducir que el camarón de roca creció desde más o menos $5.5 \mathrm{~cm}$ hasta más o menos $7.5 \mathrm{~cm}$ entre febrero $y$ agosto - sea aproximadamente $2 \mathrm{~cm}$. La diferencia entre los tamaños móximos de los individuos grandes es de $2.5 \mathrm{~cm}$ que confirma la existencia de un promedio de crecimiento de más o menos $2 \mathrm{~cm}$ cada seis meses.

En agosto se nota una moda secundaria de $4.5 \mathrm{~cm}$ y ésta parece corresponder a un segundo grupo de camarones de tamaños menores de más o menos $5.5 \mathrm{~cm}$; los camarones más pequeños fueron de $1.5 \mathrm{~cm}$ lel individuo más pequeño que se midió fue de $16 \mathrm{~mm}$.).

Esta observación nos sugiere que están presentes dos generaciones del cama- 


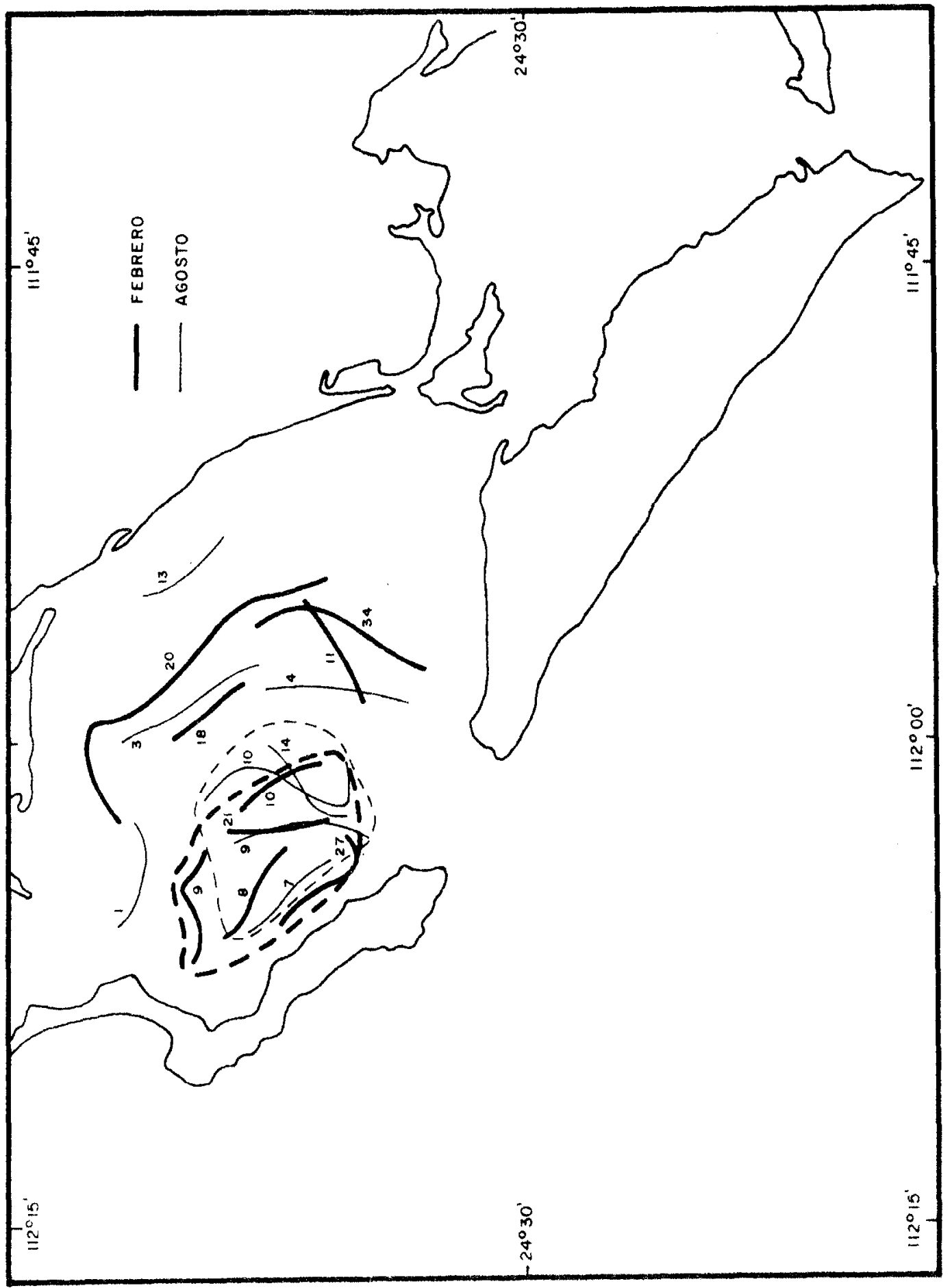

Fig. 1. Distribución del camarón de roca scyonia up en febrero y en agosio 1974. Las lineos sólidos indican los lances efectuados. Las lineas interrumpidas indicon las greas durante los dos periodos de muestreo que contienen la parte más permanente de la población 


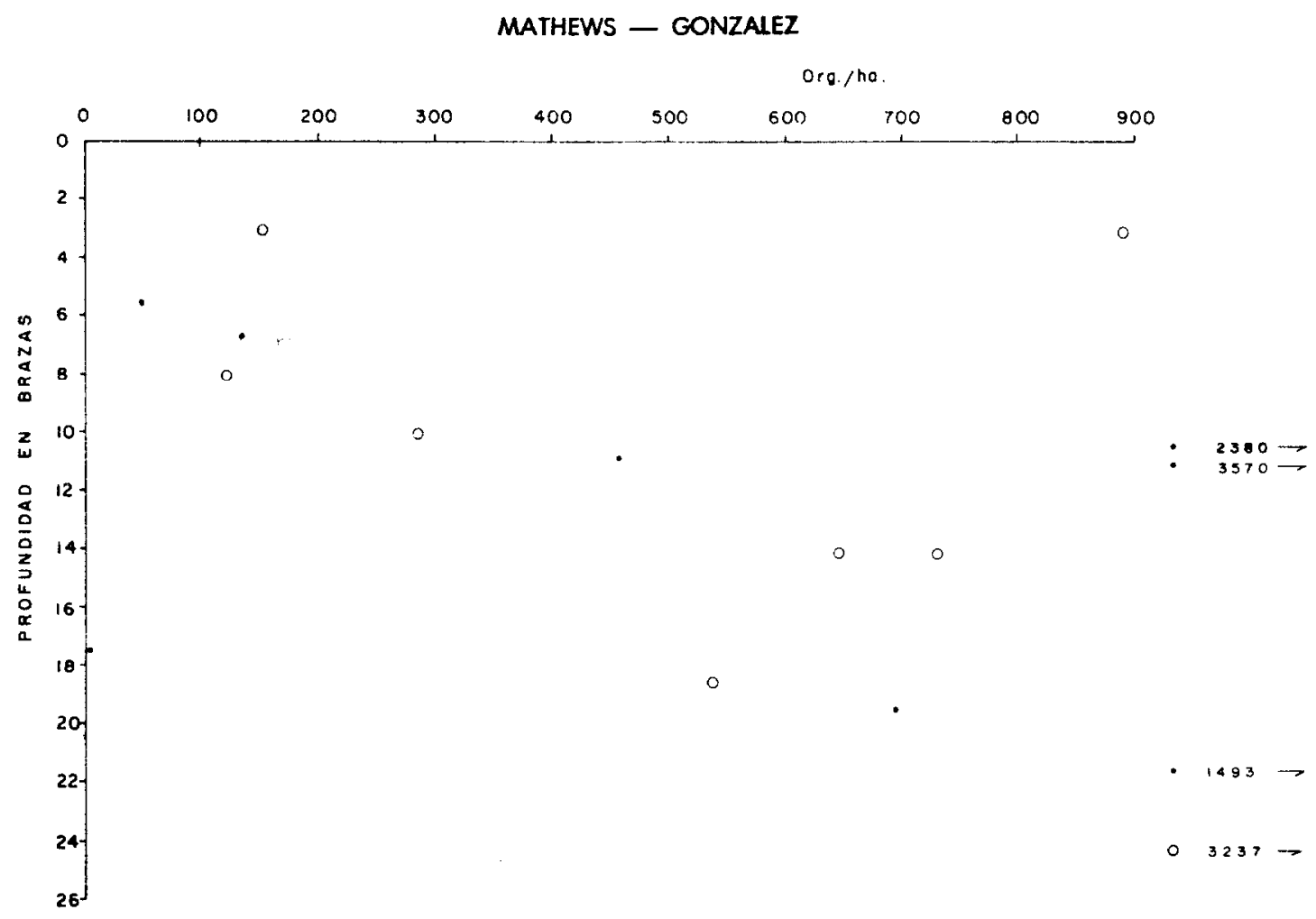

Fig. 2. Distribución batimétrica del camarón de roca, Scyonia sp, en número de individuos por ha: $O$ lances de dia; lances de noche.

rón de roca en agosto, una que estuvo presente en febrero (tamaño modal de $5.5 \mathrm{~cm}$ ) y que crecieran hasta los $7.8 \mathrm{~cm}$ en agosto $y$ una segunda generación de aproximadamente $3-5 \mathrm{~cm}$ que estuvo ausente en febrero. Esta segunda generación será la nueva generación resultante del desove que se supone ocurre entre febrero y agosto, quizá en mayo o junio. Si esta interpretación es correcta, el ciclo vital del camarón de roca sería en $\mathrm{Ba}$ hía Magdalena, aproximadamente:

(i) Desove en primavera (imayo? ijunio?l.

(ii) Crecimiento hasta $3-5.5 \mathrm{~cm}$, tamaño modal $4-4.5 \mathrm{~cm}$ para agosto ledad 3-4 meses).

(iii) Crecimiento hasta un tamaño modal de $5.5 \mathrm{~cm}$ para febrero siguiente (edad 9 meses).

(iv) Crecimiento hasta un tamaño modal de $7.5 \mathrm{~cm}$ para agosto siguiente (edad 15 meses) o sea su segundo verano.
La presencia de individuos de 9-10.5 $\mathrm{cm}$ en febrero y de 10.5 a $13.0 \mathrm{~cm}$ en agosto podría deberse a grandes diferencias en las tallas de crecimiento. Podría también deberse a la presencia de una generación más grande y en ese último caso el ciclo vital seguiría así:

(v) Crecimiento hasta 9-10.5 cm en el segundo invierno (febrerol edad más o menos de 23 meses.

(vi) Crecimiento hasta $10.5-13.0 \mathrm{~cm}$ en su segundo verano, con una edad de 29 meses.

Antes de llevar nuestro análisis a un nivel más elevádo será necesario colectar más datos sobre la especie. Concluimos provisionalmente que el camarón de roca llega hasta 15 meses de edad; seguramente llegará a una edad mayor, puesto que una muerte de toda la parte grande de la población (mayor a $7.0 \mathrm{~cm}$.) a fines de agosto es improbable. Es posible que el camarón de roca de una pequeña parte de la población llegue hasta 


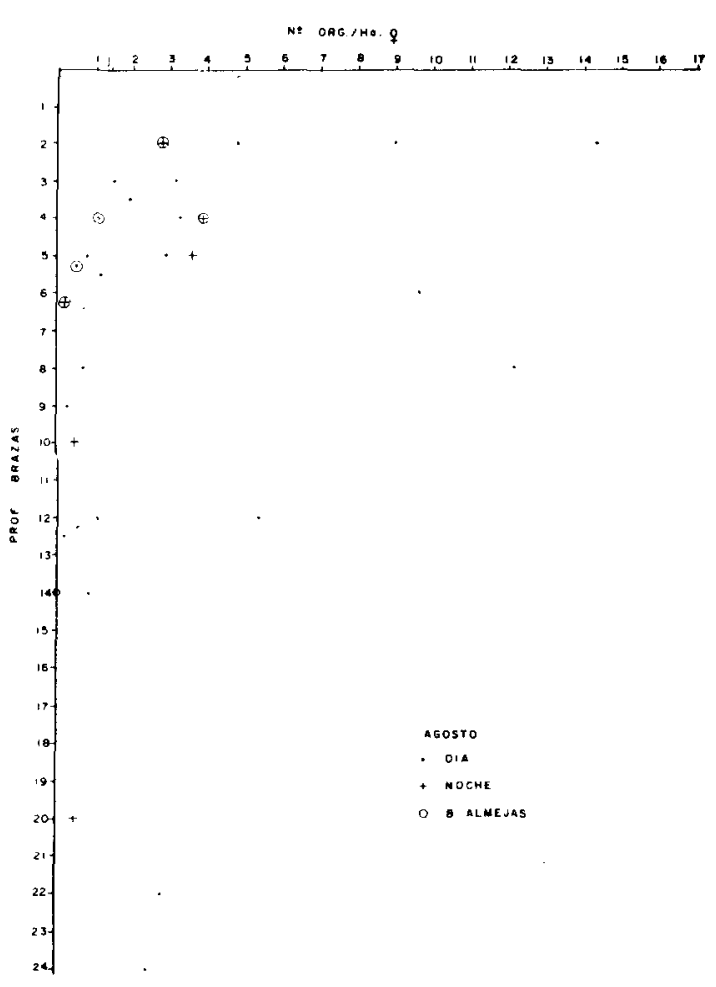

Fig. 3. Curvas de frecuencia y de longitud total de las capturas de camarón de roco, Scyonia sp.

$+\ldots+$ Febrero 1974
$+\ldots \ldots \ldots \ldots . .$.
+ Agosto 1974

30 meses de edad; en este caso los individuos más grandes y viejos 110.5 a $13 \mathrm{~cm}$.) se encuentran en agosto $y$ desaparecen antes de llegar a su tercer invierno; o sea antes del febrero siguiente, puesto que los individuos más grandes encontrados en febrero fueron de $10.5 \mathrm{~cm}$.

\section{COMPARACION SEXUAL DE LA POBLACION}

La Fig. 4 presenta las gráficas de frecuencia y tallas para los dos sexos tomadas de una muestra del camarón de
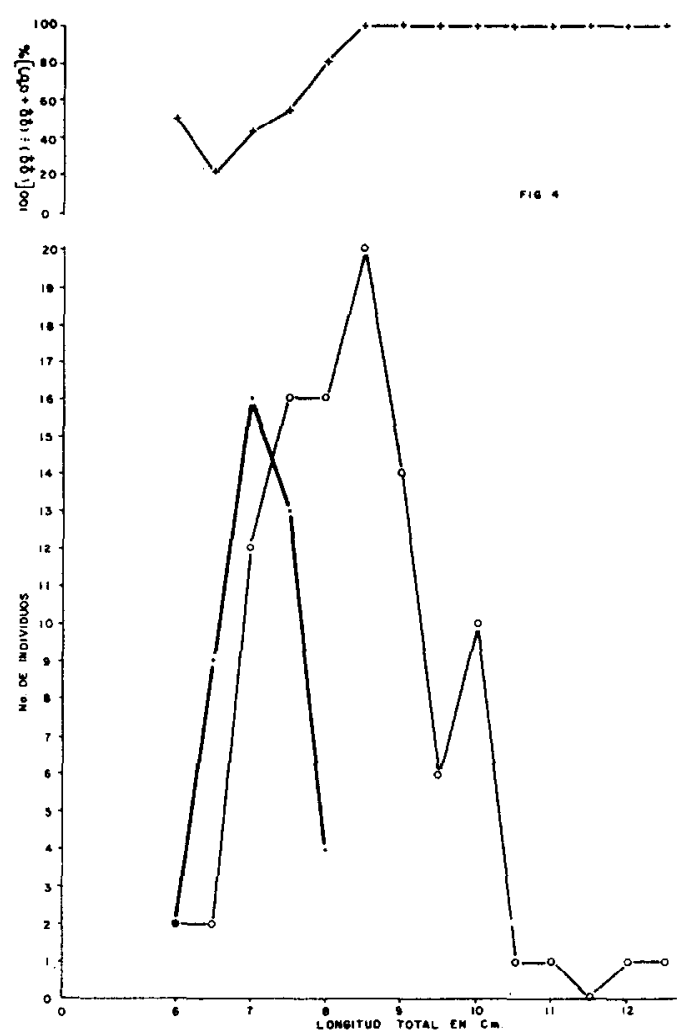

Fig. 4. Relación de sexo a longitud total .......... Número de machos o____._. Número de hembras. La gráfica superior indica la variación de la tasa sexual. definida como $\left[(q q) \div\left(q q+0^{x} 0^{x}\right)\right] \times 100$ - sea, el porciento de los camarones de roca que son hembras, siando de un intervalo de langitud particular.

roca. Se nota que los machos no exceden a los $8.0 \mathrm{~cm}$. La Fig. 3 también presenta la relación de los dos sexos, calculando según la ecuación:

(Número de hembras) $\times 100$

Número de machost = tasa sexual número de hembras) 
Cambia esta tasa de aprox. 50\% para canorones de $6-7 \mathrm{~cm}$ hasta el $100 \%$ para comarones de $8.5 \mathrm{~cm}$. para arriba. No se ilentificaron sexos de camarones menores a los $6.0 \mathrm{~cm}$., puesto que hubo una gran dificultad en determinar su sexo en las condiciones a bordo del barco. Esto nos indica que posiblemente la maduración sexual ocurre en individuos de $5-6 \mathrm{~cm}$. Este tamaña coincide de una manera general con la moda de los camarones capturados en febrero (mós o menos $5.5 \mathrm{~cm}$ ), y podemos concluir provisionalmente que los camarones desovan poco después de llegar a este tamaño y en una edad de más o menos 1.0 años.

\section{REIACION ENTRE PESO TOTAL $Y$ LONGITUD TOTAL}

En le Fig. 5 se presentan las eurvas de longitud total y peso total para el camaron de roca. Se juntaron los datos para morhos y hembras, puesto que un escruitio de los datos y un análisis preinmerir demostraron que no hubo variación del peso con el sexo. Se nota una diferencia entre los puntos para los datos de invierno $y$ verano; esto probablemente no se deba a una diferencia de condición, sino a una falla técnica: no pudimos pesar el camarón fresco en febrero y tuvimos que pesar camarón congelccio, mientras que el camarón estudich en agosto se pesó fresco. El camaror coscongelado era muy suave $y$ hasta chidratado y esto podría explicar corricncia para que el peso total de los camurones de una longitud particum c. en menor en febrero que en agosto. Se rata an la Fig. 5 un detalle que confirmu hich hipótesis. Si se debiera la diteremcin un cambio estacional de condicim, divergencia entre los datos invernetes $y$ de verano debería ser más grande pra los individuos más grandes y cextunente maduros. De hecho la ditancia as mayor para los individuos …e pequeños. Precisamente son los más pecuehos cuyos carapachos son más suawe tot vez debido a la descongelación.

\section{TAMANO DE LA POBLACION}

i) Grea ocupasa per a población per. rano es relativamente pequena, consistiendo en el área más cercana a la Isia Magdalena. Esta área contiene la mayoría de la población de verano. Se calculó el área por planimetría y es de más - menos 10,000 ha. Se ha dicho que la población de invierno no se muestreó con la precisión necesaria para delimitar el ćrea de distribución, pero se sabe que el camarón de roca fue un elemento importante sino dominante en las capturas en gran parte de Bahía Magdalena, y se asume que del área total de Bahía Magdalena de más o menos 70,000 ha., aprox. 40,000 ha fueron ocupadas por el camarón de roca. Esir cifra aunque aproximada, corresponde a la realidad observada durante la pesca.

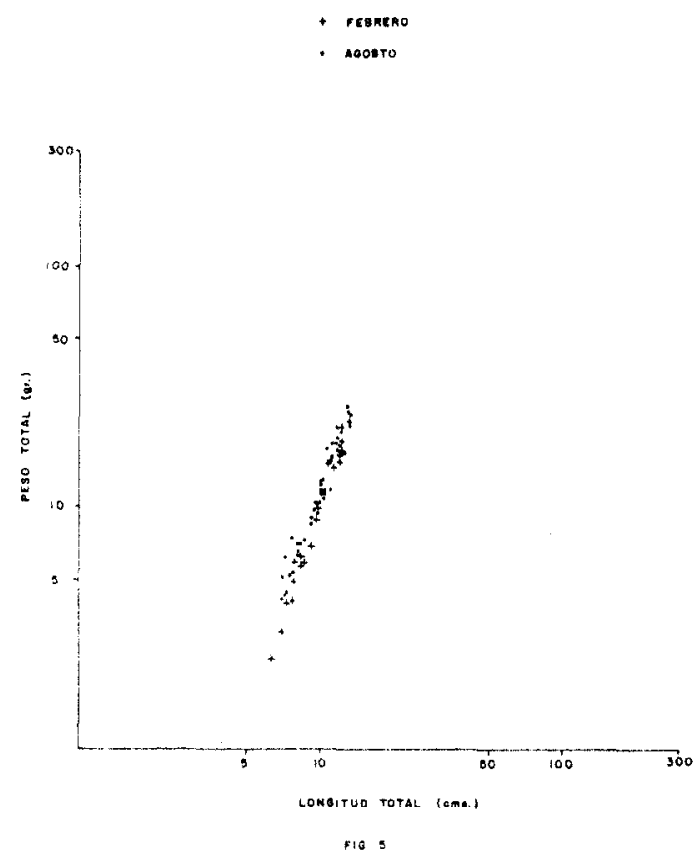

Fig. 5. Relación entre peso totol (g) y longitud total $[\mathrm{cm})$ del camarón de roca Scyonia sp.
+ Febrero

Las abundancias para invierno y verano fueron de 1099 y 852 organismos por hectćrea respectivamente; no es seguro que esas diferencias san significativas, por la gran variación que existe en los datos. Asumiendo que es válida la difereria, se calcula el tamaño de las robuciones $y$ se presenton ho resultodos 
TABLA 1

TAMAÑO APROXIMADO DE LAS POBLACIONES DE CAMARON EN ROCA

$\begin{array}{cccc}\text { Area ocupada } & \begin{array}{c}\text { Abundancias } \\ \text { (ha) }\end{array} & \begin{array}{c}\text { Población } \\ \text { (promedios or. } \\ \text { ganismos/ha) }\end{array} & \begin{array}{c}\text { Numero de } \\ \text { organismos }\end{array} \\ \text { Febrero } & 40,000 & 1,100 & 44,000,000 \\ \text { Agosto } & 10,000 & 850 & 8,500,000\end{array}$

Estos datos demuestran una mortalidad severa que acompaña a la restricción de la zona de distribución del camarón de roca, entre invierno y verano. Esta mortalidad se presenta principalmente en los camarones de 9-15 meses 15.0$8.0 \mathrm{~cm}$. en la Fig. 3); dadas las aproximaciones efectuadas en el cálculo del área de las poblaciones en invierno y verano, pensamos inapropiada una estimación del valor de la mortalidad.

Las abundancias observadas por el camarón de roca corresponden a valores de $3-11$ con un promedio de $5 \mathrm{~kg} / \mathrm{ha}$. Esto corresponde a un valor de doscientos $y$ de 50 toneladas métricas respectivamente en febrero y agosto, pero para aprovechar esta población comercialmerite será necesario efectuar la pesca en el verano, cuando la población contiene más individuos grandes pero una biomasa menor.

\section{DISCUSION}

Los datos presentados aquí se confirmarán mediante los estudios posteriores que se están llevando a cabo en la zona de investigaciones. Asimismo se llevará a cabo el análisis a un nivel más elevado; las conclusiones presentadas son preliminares; puesto que el último crucero no se llevó a cabo hasta fines de agosto y el análisis de los datos existentes no está completo. Tomando las precauciones necesarias, podemos concluir que los datos aqui presentados permi- ten un conocimiento mós preciso sobre el ciclo vital del camarón de roca. Entre los problemas que quedan por resolver mediante obtanción de datos y mós anólisis, son las tasas precisas de crecimiento y de mortalidad y los cambios exactos en la distribución estacional. Provisionalmente concluimos que el ciclo vital del camarón parece ser esencialmente similar a los ciclos de los otros peneidos de interés comercial estudiada por el Biol. Daniel Lluch Belda.

\section{AGRADECIMIENTOS}

Quiero agradecer la cooperación de los pescadores de la Cooperativa "Isla Macapule" de Guasave, Sin y al Capitán Miguel Evaristo Cárdenas del barco Macapule I. También agradecemos la cooperación de la Secretaría de Recursos Hidróulicos con quien se efectuaron estos trabajos bajo contrato con la Dirección de Acuacultura. También agradecemos la ayuda que nos prestó el P.O. Javier Amador Buenrostro Residente de la Dirección de Acuacultura en La Paz, B. C. Sur, y al Ing. Sonin Pérez Pérez Director de la Escuela Tecnológica Pesquera de San Carlos, 8. C. Sur y al Ocean. José Pérez Higuera Sub-Director de la misma. También queremos reconocer la cooperación y ayuda del Biol. Manuel Flores Villegas Jefe de Pesca en Baja California Sur; al comandante del Sector Naval de Puerto Cortés, B. C. Sur ViceAlmirante Argudín, y a todos los otros oficiales del Gobierno Federal que nos facilitaron los trabajos en esas zonas.

\section{BIBLIOGRAFIA}

Alvarez Borrego Saúl y L. Galindo Bect. Hidrologia de Bahla Magdalena, B. C. S. a fines de primavera. No publieado.

Berry, R. J. 1967. Dynamics of the Tortugas (Florida) pink shrimp populations. Ph. D. Thesis, Univ. Rhode island. Univ. Microfilms, Ann Harbor, Mich. 160 p.

Gotshall, W. D. 1972. Populations Size, Mortailty rates, and Growth rates of Northern California Ocean Shrimp, Pandalus jordoni 1965 through 1968 . St. of Calif. Dep. of Fish and Game. Fish Bulletin 155. 